\title{
COVID-19 in Patients with Hematologic Malignancies: Outcomes and Options for Treatments
}

\author{
José Carlos Martínez ${ }^{\mathrm{a}}$ R. Alejandro Sica ${ }^{\mathrm{b}}$ Keith Stockerl-Goldstein ${ }^{\mathrm{c}}$ \\ Samuel M. Rubinstein ${ }^{a}$ \\ aDivision of Hematology, University of North Carolina Chapel Hill School of Medicine, Chapel Hill, NC, USA; \\ ${ }^{b}$ Albert Einstein College of Medicine, Montefiore Medical Center, Bronx, NY, USA; 'Division of Oncology, \\ Washington University School of Medicine, St. Louis, MI, USA
}

\begin{abstract}
Keywords
Coronavirus disease $19 \cdot$ Hematologic malignancies · Outcomes
\end{abstract}

\begin{abstract}
Patients with hematologic malignancies are particularly vulnerable to infections due to underlying humoral and cellular immune dysfunction, cytotoxic chemotherapy regimens, advanced age, and the presence of comorbid conditions. Infection from severe acute respiratory syndrome coronavirus 2 , the causative agent of the COVID-19 pandemic, has become a leading cause of death globally and has disproportionally affected this high-risk population. Here, we review the cumulative evidence demonstrating worse outcomes for patients with hematologic malignancies when compared to patients with solid tumors and the general population. We examine risk factors shared with the general population (age, sex, comorbid conditions, and race) and those that are cancer-specific (cytotoxic chemotherapy, progressive disease, and cancer type), all of which confer an increased risk of severe COVID-19. Despite the historical exclusion of cancer patients from COVID-19 therapy trials, we review the emerging evidence that patients with hematologic malig-
\end{abstract}

nancies benefit from specific treatments such as convalescent plasma. Although COVID-19 vaccines are significantly less effective in this patient population, encouraging results are observed in a subset of these patients after receiving a booster dose.

(c) 2022 S. Karger AG, Basel

\section{Introduction}

COVID-19, the disease caused by severe acute respiratory syndrome coronavirus 2 (SARS-CoV-2), was classified as a pandemic by the World Health Organization (WHO) in February 2020. As of January 2022, the COVID-19 pandemic has resulted in over 340 million reported cases globally, with about 5.5 million associated deaths [1]. Here, we review the evidence that patients with hematologic malignancies have a relatively high risk of SARS-CoV-2 infection and related morbidity and mortality. Although risk factors associated with severe COVID-19 are highly prevalent in cancer patients, hematologic malignancies are independently associated with worse outcomes in COVID-19. Patients with hematologic malignancies are uniquely at risk by having underlying
Karger@karger.com www.karger.com/aha
(C) 2022 S. Karger AG, Basel

Karger
Correspondence to:

Samuel M. Rubinstein, samuel_rubinstein@med.unc.edu 
immune deficiency and receiving treatments that confer significant immunosuppression, thus challenging providers when making decisions regarding timing and the type of cancer-specific treatments. Given the underrepresentation of patients with hematologic malignancies in COVID-19 therapeutic trials and the unique needs of this vulnerable population, this group requires a targeted therapeutic approach. We address these questions by reviewing the available literature and providing recommendations based on our findings.

\section{COVID-19 Outcomes in Patients with Hematologic Malignancies}

The first studies from the initial epicenter of the COVID-19 pandemic in China found an overrepresentation of cancer patients in those infected with SARS-CoV-2 and worse outcomes for those requiring hospitalization $[2,3]$. Compared to patients in the general population, patients with cancer and COVID-19 had an increased risk of severe COVID-19 (defined by admission to the ICU or death) at $39 \%$ versus $8 \%$ [2] and $53.6 \%$ versus $4.7 \%$ (with $28.6 \%$ versus $2.3 \%$ mortality) [3]. A limitation shared by early studies and reports was the small number of cancer patients and the scarce representation of cancer subtypes in cohorts. The first large cohort of cancer patients with COVID-19 recapitulated these initial findings by showing that cancer patients with COVID-19 had higher mortality (OR 2.34, 95\% CI: 1.15-4.77) and a higher ICU admission rate (OR 2.84, 95\% CI: 1.59-5.08) when compared to patients without cancer [4]. Interestingly, it found that patients with hematologic cancers (including leukemia, lymphoma, and multiple myeloma) had the highest death rate $(33.33 \%)$ and ICU admission rate (44.44\%) of any population of patients with cancer. However, the number of patients with hematologic malignancies in this cohort was small. These findings were replicated in epicenters outside of China as the pandemic spread. A study from the first wave in New York City reported a similarly high mortality rate at $28 \%$ in a large cohort of cancer patients with COVID-19 [5]. Notably, mortality was higher in patients with hematologic malignancies (37\%) than those with solid malignancies (25\%).

The first studies reporting outcomes in dedicated large cohorts of patients with hematologic malignancies came from Italy and Spain $[6,7]$. In a cohort of 536 patients with hematologic malignancies and COVID-19, with a median follow-up of 20 days, overall mortality was $37 \%$, nearly a 2 -fold increase in mortality (HR: $2.04,95 \% \mathrm{CI}$ :

COVID-19 in Patients with Hematologic

Malignancies
1.77-2.34) compared to the general population of Italian patients with COVID-19 [6]. Additionally, among patients with hematologic malignancies, there was a 41 -fold increase in mortality of those with COVID-19 compared to those without COVID-19 (41.3, 95\% CI: 38.1-44.9). A caveat of this early study is a likely overestimation of mortality due to an overrepresentation of hospitalized patients and low ICU utilization rate, suggesting a bias toward enrollment of patients with severe disease and rationing of healthcare resources [8]. A similarly high rate of severe disease and mortality (62\% and 33\%) was observed in patients with hematologic malignancies and COVID-19 compared to the general Spanish population with COVID-19 (15\% and 10\%) [7]. The authors noted that while most patients required hospitalization (87\%) and most deaths occurred in hospitalized patients, only $8 \%$ were admitted to the ICU.

To respond to the new challenges presented by the pandemic, international groups of clinicians and scientists created several patient registries [9]. One such registry, the COVID-19 and Cancer Consortium (CCC-19), originated early in the pandemic on social media, utilizing a REDCap-based survey tool to compile real-time observational data on the outcomes of patients with cancer and COVID-19 at scale $[10,11]$. An analysis of the first 1,000 patients in the CCC- 19 registry revealed that $26 \%$ of patients in this cohort experienced a composite endpoint of severe COVID-19 (including death, ICU level of care, mechanical ventilation, and severe illness requiring hospitalization) and had a 30-day mortality of $13 \%$ [10]. Patients with hematologic malignancies were found to be at increased risk of severe COVID-19 (adjusted OR 1.77, 95\% CI: 1.23-2.56). Over time, as the CCC-19 cohort grew, analyses demonstrated increased COVID-19-specific mortality for patients with hematologic malignancies as well [12]. Other registries replicated this finding [13-16]. Specifically, the UK Coronavirus Cancer Monitoring Project was among the first registries to report on the association between tumor type and outcomes in a large cohort $(1,044)$ of cancer patients with COVID-19 [13]. In their cohort, they found overall mortality of $30.6 \%$, with more severe COVID-19 in patients with hematologic malignancies (leukemia, lymphoma, and myeloma) when compared to those with solid tumors (OR $1.57,95 \%$ CI 1.15-2.15). Only leukemia was associated with significantly higher mortality (OR 2.25, 95\% CI: 1.13-4.57). Outcomes of the aforementioned studies are summarized in Table 1.

Unique among patient registries is the American Society of Hematology Research Collaborative (ASH RC) 


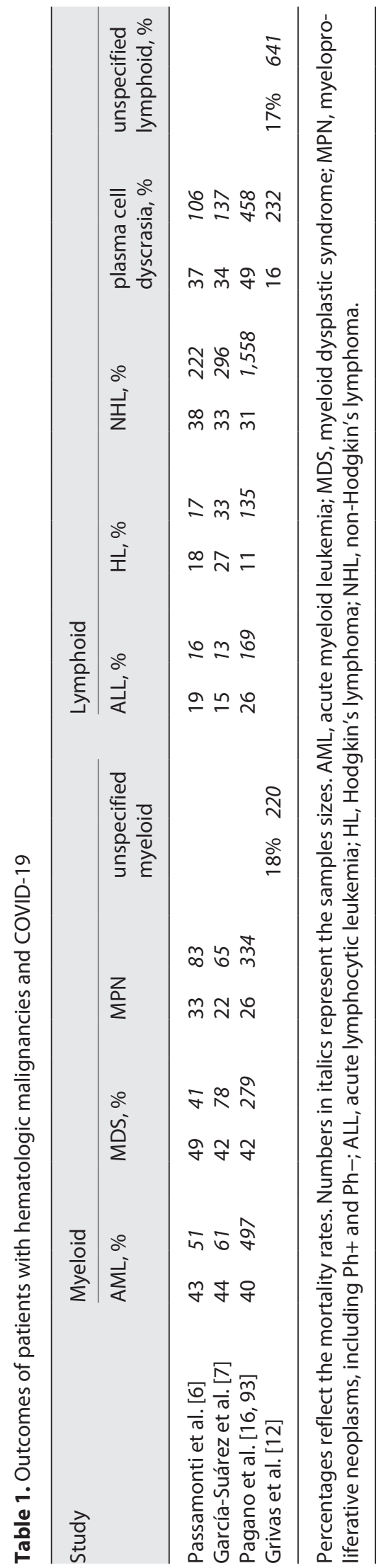

COVID-19 registry, which provides real-time data in a dashboard, reporting outcomes in patients with COVID-19 and hematologic conditions (including nonmalignant) across the globe [17]. The authors observed a mortality rate of $28 \%$ and $42 \%$ in those hospitalized in their report on the outcomes of the first 250 patients [18]. Interestingly, they noted that $16 \%$ of patients declined ICU care in lieu of palliative measures (most of whom died). In contrast, almost half of the hospitalized patients with a physician-estimated prognosis of more than 12 months who received maximal supportive care survived. Unfortunately, no studies have yet reported the context in which decisions to admit patients with COVID-19 and hematologic malignancies to an ICU are made, and such data are difficult to collect in registries.

These data need to be contextualized in the setting of global trends in care and outcomes for patients with cancer and COVID-19. Early in the pandemic, it seems plausible that patients with cancer may have been disproportionately denied access to the appropriate level of care due to limited ICU resources, which necessitated rationing of care. The first systematic review and meta-analysis of patients with cancer and COVID-19 admitted to the ICU showed an overall mortality of $60.2 \%$ with an increased risk among those with hematologic malignancies (OR 1.88, 95\% CI: 1.17-3.01) [19]. However, the authors point out that this mortality rate is not prohibitively high to automatically bar admission to an ICU. Despite this, several studies showed a relatively low ICU admission rate despite the high mortality rate of patients with COVID-19 and hematologic disease $[6,7,10,18,20]$. This evidence suggests that this patient population may have been deprived of proper resource allocation, at least early in the pandemic. Despite high mortality for patients with hematologic malignancies, they should not be disqualified from receiving intensive treatment when appropriate.

In addition to disposition, the overall outcomes of patients with cancer and COVID-19 have improved since the first outcomes studies [16]. In the early phases of the pandemic, little was known about best practices for treating COVID-19. Patients diagnosed with COVID-19 during this time did not have access to therapies that were later found to have benefit, such as dexamethasone, remdesivir, and therapeutic monoclonal antibodies. Additionally, unproven treatments such as hydroxychloroquine were widely used off label; in some studies, such as the first CCC-19 study, such therapies were associated with worse outcomes for patients with cancer and COVID-19. New reports from the CCC-19 group suggest that cancer patients recently diagnosed with COVID-19 
have better outcomes than earlier during the pandemic [12]. Despite this trend of improved outcomes, the relatively increased risk of severe COVID-19 seen in patients with cancer has persisted. There remains an urgent unmet need to comprehensively understand the root causes of increased mortality to optimize mitigation strategies for this vulnerable population. In sum, numerous studies have consistently demonstrated that patients with hematologic malignancies experience higher rates of severe COVID-19 than control groups of patients with and without cancer.

\section{Noncancer-Related Risk Factors}

The etiology of the increased risk of severe COVID-19 in patients with hematologic malignancies is likely multifactorial and may include factors unrelated to the primary malignancy. Patients with hematologic malignancies are disproportionately likely to have noncancer-related risk factors for severe COVID-19, such as older age and male sex. Data from the US Surveillance, Epidemiology, and End Results registry report a median age-group at diagnosis of 65-74 years old for patients with acute myeloid leukemia (AML), MM, and CLL with male to female ratios of $1.4,1.5$, and 1.9, respectively [8]. The risk of severe COVID-19 increases above the age of 40 years, with monotonic increments in COVID-19 severity with increased age [6]. Pediatric patients with cancer and COVID-19 seem to fare significantly better, with $4 \%$ mortality for those less than 18 years of age compared to $34 \%$ for those older than 60 years of age [21]. Since older age is consistently associated with worse COVID-19 outcomes, the advanced age of many patients with hematologic malignancies increases their baseline risk of severe $\mathrm{CO}$ VID-19.

Multiple studies have identified male sex as a factor associated with worse COVID-19 outcomes in both the general population and patients with cancer. For example, a large meta-analysis including 90 studies with over 3 million cases reported that males had a higher likelihood of ICU admission (OR $=2.84,95 \%$ CI: 2.06-3.92) and higher risk of death $(\mathrm{OR}=1.39,95 \% \mathrm{CI}$ : $1.31-1.47)$ compared to females [22]. Similarly, patients with poor performance status, generally defined as an ECOG performance status greater than or equal to 2 , have worse outcomes than patients with ECOG performance statuses of 0 or 1 [10, 12, 23-25]. In addition, comorbidities such as obesity, cardiovascular disease, pulmonary disease, renal disease, and diabetes mellitus are also risk factors for severe COVID-19 in patients with cancer [12]. Out of these, renal disease appears to be more consistently associated with worse outcomes in cohorts of patients with hematologic malignancies and COVID-19 [26, 27].

COVID-19 severity has been linked to the degree of inflammation and subsequent immune response, with cytokine storm leading to worse outcomes $[28,29]$. This has led to the development of predictive models that incorporate markers of inflammation, immune, and organ function [30]. Although there are no reports on the predictive value of such biomarkers specifically in patients with hematologic malignancies, several studies have replicated the findings observed in the general population of patients with cancer [12, 24, 25]. Abnormal biomarkers of inflammation and immune dysregulation have been associated with increased rates of severe COVID-19. These include C-reactive protein, interleukin-6 (IL-6), TNF-alpha, ferritin, and D-dimer. In addition, abnormalities in the complete blood count, including low platelets, low or high absolute lymphocyte count, high absolute neutrophil count, and increased neutrophil-to-lymphocyte ratio, have been associated with worse COVID-19 outcomes [31]. As patients with hematologic malignancies have complete blood count abnormalities associated with their diseases, the independent effects of complete blood count abnormalities and hematologic malignancies on COVID-19 outcomes are not well-understood. Lastly, markers of tissue damage, such as serum creatinine and troponin, may reflect irreversible deficits due to COVID-19 and are associated with increased COVID-19 severity in patients with cancer [12].

Reports early in the pandemic raised concerns for a disproportionate impact of COVID-19 on racial and ethnic minorities [32, 33]. Some hematologic malignancies are more common in racial and ethnic minorities, such as multiple myeloma in Black patients and acute lymphoblastic leukemia in Hispanic patients. These disparities may contribute to the increased COVID-19 severity seen in patients with hematologic malignancies. A systematic review reported disproportionally higher rates of SARSCoV-2 infections and COVID-19-related mortality among Black and Hispanic patients [34]. The authors identified decreased access to healthcare and increased exposure risk as likely contributing to this disparity. Among patients with cancer, the COVID-19 and Cancer Outcomes Study (CCOS) found that Black and Hispanic patients had higher infection rates compared to White patients [35]. They also reported a decreased utilization rate of telehealth visits among minorities and increased treatment delays among Hispanic patients. Early data 
from the CCC-19 reported that Black patients with cancer and COVID-19 were half as likely to receive remdesivir, a therapy that has shown a modest benefit in patients hospitalized with severe COVID-19 [36]. An updated report from the CCC-19 noted that non-White race/ethnicity was associated with higher COVID-19 severity and that non-Hispanic Black patients had higher 30-day mortality [12]. Among patients with hematologic malignancies, an early report on patients with multiple myeloma and COVID-19 identified race and ethnicity as the most significant risk factors for worse outcomes among those hospitalized [37]. In a systemic review and meta-analysis of patients with hematologic malignancies, non-White patients had an estimated 2-fold higher risk of death from COVID-19 when compared to White patients (RR 2.2, 95\% CI: 1.3-3.8) [21].

\section{Cancer-Specific Risk Factors}

The increased prevalence of noncancer-related risk factors for severe COVID-19 in patients with hematologic malignancies is critical. However, disease-related factors, such as cancer status, cancer type, stage, and treatment history, play a significant role in the increased risk of severe COVID-19 experienced by patients with hematologic malignancies [38]. The first systematic review and meta-analysis of outcomes in patients with hematologic malignancies and COVID-19 described these disparities [18]. The authors found an overall case mortality rate of $34 \%$ (95\% CI: $28-39 \%, N=3,240)$. However, they found differences in outcomes among different cancer types, with patients with acquired bone marrow failure and acute leukemia having the highest mortality rates at 53\% and $41 \%$, respectively. Using multivariable Cox regression analysis, AML (adjusted OR 3.49, 1.56-7.81), indolent non-Hodgkin lymphoma (adjusted OR 2.19, 95\% CI: 1.07-4.48), aggressive non-Hodgkin lymphoma (adjusted OR 2.56, 95\% CI: 1.34-4.89), and plasma cell neoplasms (adjusted OR 2.48, 95\% CI: 1.31-4.69) were found to be associated with worse outcomes than a control population of patients with chronic myelogenous leukemia [6]. Several other studies demonstrate a worse prognosis for patients with AML and COVID-19 when compared to patients with hematologic malignancies as a whole [7, $13,16]$. It is unclear why patients with AML appear to have worse outcomes. However, these patients often have an increased risk of infections due to prolonged periods of impaired innate and adaptive immunity that is malignancy and treatment-related. In addition, due to the ag- gressive biology of disease, these patients may have higher disease-related mortality in the setting of holding therapy to allow convalescence from COVID-19. It would be helpful for future studies to tease out the differences between COVID-19-related mortality and overall mortality in this group to better assess the specific mortality risk attributed to COVID-19.

The biologic mechanisms by which hematologic malignancies confer increased risk for worse outcomes in COVID-19 have not been fully elucidated. The degree of immune dysfunction associated with hematologic malignancy is disease-specific and is influenced by the disease stage and type of cancer-directed therapy. Emerging evidence suggests that dysregulated humoral and cellular immune response to SARS-CoV-2 in patients with cancer could partly explain the differences in outcomes [39]. Corroborating prior studies, patients with hematologic malignancies had higher overall mortality when compared to patients with solid tumors ( $55 \%$ vs. $33 \%, p=$ $0.075)$. Interestingly, the authors found that the immune phenotypes associated with SARS-CoV-2 infection were similar in patients with solid tumors and in those without cancer. Importantly, patients with hematologic malignancies had significant B-cell and humoral immunity defects but appeared to have better outcomes when able to mount an appropriate CD8+ T-cell response. Patients with hematologic malignancies (including AML, MM, and CLL) have features of T-cell dysfunction (senescence and exhaustion) at diagnosis [40-42]. However, the degree of T-cell dysfunction and response to COVID-19 among patients with the various hematologic malignancies has not been thoroughly characterized. It would be interesting for future studies to assess whether such differences contribute to the observed variation in outcomes, for example, whether the increased T-cell dysfunction seen in AML contributes to worse COVID19-specific outcomes.

Despite a beneficial compensatory T-cell response in patients with cancer and COVID-19, there is an association between increased mortality and blunted humoral immune response [39]. This implicates an essential role for B cells and derived antibody response in SARS-CoV-2 infection. Other studies have reported that a coordinated SARS-CoV-2-specific adaptive immune response, which diminishes with aging, is associated with less severe $\mathrm{CO}$ VID-19 [43]. Therefore, patients with B-cell malignancies are particularly at risk of severe COVID-19 due to compromised B-cell function and advanced age (median age of 70 years at diagnosis). Patients with hematologic malignancies receiving cellular therapies (stem-cell transplant 
and CAR-T cells) also appear especially susceptible to COVID-19 due to the significant depth and duration of immune suppression associated with these treatments. An early study out of Memorial Sloan Kettering looking at the outcomes of patients with COVID-19 after receiving cellular therapies showed an overall survival of $78 \%$ at 30 days [44]. The authors noted that active malignancy such as relapsed leukemia drove mortality in their cohort. Reports from the Center for International Blood and Marrow Transplant Research (CIBMTR) and the European Society for Blood and Marrow Transplantation (EBMT) show an overall survival in patients undergoing allogeneic stem-cell transplant (allo-SCT) at $68 \%$ and $78 \%$, respectively, and in those undergoing autologous stem-cell transplant at $67 \%$ and $72 \%$, respectively $[45,46]$. Concerningly, the mortality rate in those receiving CAR-T cells has been recently reported to be substantial $[16,47]$. In a large European cohort of CAR-T cells patients with COVID-19 infection, $66.7 \%$ of patients had severe disease, $43.3 \%$ required ICU admission, and the COVID-19-related mortality was 33\% [47]. Altogether, these data suggest that despite the immunosuppression associated with cellular therapies, the mortality in this group of patients is likely driven by the underlying disease state.

Patients with progressive cancer appear to be at exceptionally high risk of poor outcomes in the setting of active COVID-19, across several studies $[4,10,12,14,24]$. This finding has held in populations of patients specifically with hematologic malignancies [6, 20, 23, 26]. In general, these studies report a 2-3-fold increased risk in overall mortality associated with active or progressive malignancy. This association is likely multifactorial. Hematologic malignancies themselves impair humoral and cellular immunity, and patients with hematologic malignancies experience disease-related immunosuppression. In addition, these patients have often been exposed to one or more lines of antineoplastic therapy for their hematologic malignancies, which can have persistent immunosuppressive effects. As such, patients with progressive hematologic malignancies are experiencing detrimental effects of disease and treatment on the immune system simultaneously, which can contribute to worse outcomes in the setting of COVID-19.

\section{The Impact of Chemotherapy on Outcomes}

Although many cancer therapies are associated with some degree of immune suppression, the specific depth, duration, and type of immunosuppression are highly variable. To that end, studies examining the association between the recent administration of cytotoxic chemotherapy and outcomes in patients with cancer and COVID-19 have produced conflicting results. Some studies show an association between antineoplastic therapy and COVID-19 severity [12, 13, 25], and others report no association $[6,10,21,48]$. It is likely that the risk of COVID-19 is widely divergent and depends on the specific antineoplastic therapies received. To date, no study has had a sufficient sample size to investigate individual regimens and therapies appropriately. In a meta-analysis early in the pandemic, patients who received recent systemic anticancer therapy compared to no treatment had a RR of death of 1.17 (95\% CI: $0.83-1.64, N=736$ ) [21]. A lack of statistically significant excess risk of death was preserved in a subgroup analysis of patients receiving cytotoxic chemotherapy (RR 1.29, 95\% CI: 0.78-2.15). However, the authors noted significant heterogeneity regarding the definition of "recent chemotherapy" used among the analyzed studies with ranges in the timeline of chemotherapy administration of 1-3 months. In an analysis of CCC-19 data, there was a high mortality rate among patients with hematologic malignancies in those receiving R-CHOP-like systemic chemotherapy $(45 \%, n=22$, diffuse large B-cell lymphoma [DLBCL] being the most common indication) and hypomethylating agents (50\%, $n=12$, myeloid dysplastic syndrome being the most common indication) [12]. Interestingly, they found a paradoxically low mortality rate $(10 \%, n=10)$ among patients with multiple myeloma receiving combination therapy with daratumumab, immunomodulatory drugs, and steroids despite the expected high degree of immunosuppression with this regimen. However, they noted considerable heterogeneity among the cytotoxic regimens reported in the study and that no regimen was received by more than 31 patients, thus limiting the power to detect clinically and statistically significant effects. Altogether, this study demonstrates no clear relationship between the degree of immunosuppression associated with specific cytotoxic chemotherapies and their corresponding outcomes in patients with hematologic malignancies and COVID-19.

A large cohort of patients with lymphoma and COVID-19 reported a mortality rate of $34.5 \%$, with a median follow-up at 27 days [20]. Notably, the authors found that active disease was associated with a significant increase in the risk of death (HR: 2.43 , 95\% CI: 1.23-4.77), while active treatment and type of treatment were not. The authors also noted that patients with DLBCL had reduced survival compared to patients with follicular lymphoma 
(50\% vs. $80 \%$ survival, HR: 2.66 , $95 \%$ CI: $1.35-5.247$ ). A greater proportion of patients with DLBCL $(27 / 33,81.8 \%)$ were on active CHOP-like chemotherapy when compared to patients with follicular lymphoma (22/54, $40.7 \%)$. Similarly, in a cohort of patients with myeloid dysplastic syndrome and COVID-19, the reported mortality rate was very high at almost $48 \%$ [49]. Most deceased patients $(17 / 30)$ had high or very high IPSS-R prognostic scores, and as a result, the use of hypomethylating agents was overrepresented in the group of deceased patients (15/30) compared to those alive (5/33). The presence of active disease may confound these results, and these studies are as of yet underpowered to assess the independent associations of therapy and disease status with outcomes. Given the conflicting findings in the literature, there is a need for larger cohorts of subtype-specific patients with hematologic malignancies to study further the relationship between specific cancer-targeted therapies and COVID-19-specific outcomes.

Despite the unclear association between cytotoxic chemotherapy and outcomes in patients with hematologic conditions and COVID-19, some exciting reports suggest a protective effect from cancer-directed therapies [50, 51]. Specifically, COVID-19 severity has been linked to a hyperinflammatory immune response. Therefore, it has been postulated that targeting cytokine production and macrophage activation with JAK and BTK inhibitors could improve outcomes $[52,53]$. In a large cohort of patients with myeloproliferative neoplasms and COVID-19, the overall survival was reported at $28.6 \%$, with a worse prognosis among patients with myelofibrosis (48\%) [50]. Interestingly, the authors found high mortality (40\%) in the ruxolitinib treatment group. However, they found that the mortality was mainly accounted for by those who had rapid treatment discontinuation ( $75 \%$ of deaths). Using logistic regression and sensitivity analysis by propensity score matching, they determined that there was no association between ruxolitinib treatment and overall mortality, but instead, there was a significant increase in mortality associated with its discontinuation. This was not the case for hydroxyurea, the other most common treatment modality reported in the study. These results suggest a potential protective effect for ruxolitinib in patients with hematologic malignancies, although more data are needed.

Notably, in a European cohort of CLL patients with COVID-19, there was an overall significant decrease in hospitalization rate for severe COVID-19 in those on ibrutinib (OR 0.44, 95\% CI: 0.20-0.96) when compared to those on other regimens or off treatment [51]. Addi- tionally, a small case series out of the UK reported worse outcomes in treatment-naive CLL patients with COVID-19 [54]. However, another large cohort of CLL patients with COVID-19 did not show any association between the use of BTKi and outcomes, although most patients discontinued their BTK inhibitor at COVID-19 diagnosis [27]. A revised and updated report showed improved outcomes in later cohorts of patients with CLL and COVID-19 during the pandemic [55]. The authors attributed these improvements to the use of new COVID-19 directed therapies, but interestingly, they also noted a significant difference in BTKi discontinuation between the early (76\%) and later cohorts (20\%). The potential protective effects of ibrutinib have also been reported in patients with Waldenström macroglobulinemia with COVID-19, where it was noted that these patients had a milder disease course [56]. However, a phase II trial of acalabrutinib (NCT04346199) in the general population failed to reach its primary endpoint of improving mortality and respiratory failure in patients with $\mathrm{CO}$ VID-19.

In general, a consensus on the effect of antineoplastic therapy on the outcomes of patients with cancer and COVID-19 does not emerge from a review of the literature on this topic. As we have previously stated, however, progressive malignancy is a clear and robust risk factor for severe COVID-19. In general, optimizing control over the primary malignancy to the extent possible should be seen as a potential primary prevention strategy against COVID-19.

\section{COVID-19 Therapeutics in Patients with Hematologic Malignancies}

To date, several large clinical trials in the general population have reported on the effectiveness of specific COVID-19 targeted therapies. Those that have been reported to improve outcomes include dexamethasone [57], remdesivir [58], tocilizumab [59], and monoclonal antibodies (only in those who were seronegative) [60]. The following therapies have not shown any benefits in the general population of patients with COVID-19: azithromycin [61], hydroxychloroquine [62], lopinavir-ritonavir [63], and convalescent plasma [64-66]. However, patients with hematologic malignancies were often excluded from or underrepresented in these studies, making the findings difficult to extrapolate to this population. Therefore, the evidence in this setting is guided mainly by retrospective cohort studies of patients with hematologic malignancies 
and COVID-19. An early report from the CCC-19 registry showed an increase in mortality in patients with cancer receiving hydroxychloroquine in combination with other therapies and no benefit in those receiving hydroxychloroquine alone or high-dose steroids [36]. However, they reported a survival benefit for those treated with remdesivir, although not statistically significant. Similarly, an early cohort of patients with hematologic malignancy and COVID-19 showed no survival benefit from hydroxychloroquine, but it demonstrated a survival benefit from azithromycin and low-dose prednisone [23]. Remdesivir use was very limited early in the pandemic, at least in cohorts of patients with hematologic malignancies. A more recent study out of Israel reported a decreased mortality rate in patients with COVID-19 and hematologic malignancies receiving remdesivir and no benefit in those receiving steroids or convalescent plasma [67]. Although the FDA granted emergency use authorization (EUA) for two oral antiviral medications, ritonavir-boosted nirmatrelvir (NCT04960202) and molnupiravir (NCT04575597), there are no published data about the efficacy of these agents in patients with hematologic malignancies $[68,69]$.

Convalescent plasma has been proposed as a potentially effective intervention to overcome deficient humoral immunity in patients with hematologic malignancies and COVID-19. These patients have impaired seroconversion due to COVID-19 infection and COVID-19 vaccination, and the biologic rationale for using viable antibodies from a recovered donor is strong [70]. Thompson and et al. [71] conducted a retrospective cohort study on propensity score-matched patients with hematologic malignancies and COVID-19 from the CCC-19 registry. The authors found that the use of convalescent plasma was associated with a lower 30-day mortality (HR: 0.60, 95\% CI: $0.29-0.92)$ and lower mortality in those admitted to an intensive care unit $(0.4,95 \%$ CI: $0.2-0.8)$ or receiving mechanical ventilation $(0.32,95 \%$ CI: $0.14-0.72)$. Unfortunately, the effectiveness of convalescent plasma for the treatment of COVID-19, in general, has been controversial. Some meta-analyses show that this intervention is beneficial $[72,73]$, while others do not show a benefit [74, 75]. In general, the randomized trials of convalescent plasma in patients with COVID-19 have been negative [64-66]. In addition, there are methodological concerns with the studies' heterogeneity in the timing of application, case severity, dosage, and patient characteristics, with the evidence suggesting that it is most effective when used early and at high titers [72]. Moreover, patients with hematologic malignancies might represent a special

COVID-19 in Patients with Hematologic Malignancies group that benefits from this treatment modality due to their immunocompromised state at baseline. Future clinical trials with higher inclusion of patients with hematologic malignancies are necessary to address this question.

Therapeutic monoclonal antibodies have demonstrated a more consistent benefit than convalescent plasma. There have been three monoclonal antibody combinations under EUA by the US Food and Drug Administration in the USA: bamlanivimab-etesevimab (NCT04427501), casirivimab-imdevimab (NCT04992273), and sotrovimab (NCT04913675). These agents have generally shown the most benefit in outpatients at high risk of developing severe COVID-19. Given better trial results in the general population of patients with $\mathrm{CO}$ VID-19, it is plausible that the benefit of therapeutic monoclonal antibodies in patients with hematologic malignancies and COVID-19 is greater than the benefit associated with convalescent plasma. Data on this specific population, though minimal, are promising. In one single-center retrospective study, 42 patients with cancer in general and COVID-19 received therapeutic monoclonal antibody preparations, mostly bamlanivimab-based [76]. Of these, 5 (12\%) were admitted to the hospital, with only one (2\%) requiring an ICU level of care. A retrospective study of patients with mild or moderate COVID-19, 7\% of whom were immunocompromised, demonstrated a significant decrease in hospitalization [77]. Although uncontrolled, these outcomes are better than those shown in larger cohorts. Additional study of these agents in patients with hematologic malignancies is needed, including prospective studies of these agents administered in novel settings, such as high-risk exposures.

An inflammatory cytokine signature, driven by IL-2 and TNF-alpha signaling, has been implicated in SARS$\mathrm{CoV}-2$ infection severity and associated with outcomes of COVID-19 infection [29]. This led to the design of randomized clinical trials to assess the effectiveness of IL-6 antagonists, mainly tocilizumab, in reducing morbidity and mortality associated with COVID-19. Tocilizumab, a humanized recombinant monoclonal antibody that binds to membrane-bound and soluble IL-6, has been previously shown to be effective in treating CAR-T associated cytokine release syndrome [78]. Studies looking at the effectiveness of tocilizumab in decreased COVID-19 associated mortality have shown mixed results, with some reporting a benefit $[59,79,80]$ and others no benefit $[81$, 82]. A prospective meta-analysis of randomized trials showed reduced 28-day all-cause mortality with the use of IL-6 antagonists in COVID-19 [83]. A meta-analysis 
demonstrated that tocilizumab is associated with reduced 30-day mortality, ICU admissions, mechanical ventilation, and superinfection rates in patients with COVID-19 not admitted to the ICU [84]. A randomized clinical trial of tocilizumab for the treatment of COVID-19 specifically in patients with cancer and COVID-19 (NCT04370834) was stopped prematurely after only enrolling 1 patient [81]. Currently, there is one clinical trial actively enrolling patients with breast cancer and COVID-19 to assess the efficacy of tocilizumab in this specific population (NCT04871854) but none specific to patients with hematologic malignancies.

The most effective strategy to prevent COVID-19 infections, related hospitalizations, and mortality is vaccination [85]. However, emerging data suggest that patients with hematologic malignancies have decreased immunogenic response to COVID-19 immunization [86-88]. This is more pronounced in patients with B-cell malignancies, those receiving CAR-T cells and undergoing allo-SCT, and in those having received recent anti-CD20 antibodies (particularly in the 6 months before COVID-19 diagnosis) [89-91]. Notably, patients with hematologic malignancy are less likely to have a humoral immune response, albeit having a cellular immune response to vaccines, exacerbated by anti-CD20 and small-molecule therapies [92]. Interestingly, this discordant response seems to be more prevalent with BNT162b2 (BioNTech, Pfizer) and not mRNA-1273 (Moderna) [92]. Despite these challenges, a significant decrease in overall mortality has been reported for patients with hematologic malignancies and COVID-19 in the postvaccination period compared to pre-vaccination at $12.4 \%$ versus $31 \%$, respectively [93]. Recent novel developments in the use of monoclonal antibodies for pre-exposure prophylaxis in immunocompromised patients are promising. The FDA granted tixagevimab-cilgavimab (Evusheld) an EUA after the PROVENT trial (NCT04625725) and demonstrated that this agent resulted in a 77\% reduction in COVID-19 infections at 6 months [94].

\section{Conclusions}

Patients with COVID-19 and hematologic malignancies have increased mortality and more severe disease than patients with COVID-19 and solid tumors or the general population. Patients with hematologic malignancies have a high prevalence of general risk factors (older age, smoking, and comorbid conditions) associated with COVID-19 severity. However, it appears that tumor type and active and progressive disease are independently associated with worse outcomes. Partly explaining these differences in outcomes is emerging data showing humoral and cellular immune dysfunction with resulting impaired immunogenic response to SARS-CoV-2 infection in patients with hematologic malignancies. However, additional studies are needed to further characterize the immunogenic response in COVID-19 across hematologic cancer subtypes and different disease stages. This has made treatment decisions challenging as the treatment itself often results in significant immunosuppression. In addition, there are conflicting data regarding the association between recent chemotherapy administration and outcomes in COVID-19. This is likely due to the significant heterogeneity of clinical settings and relatively small numbers of patient cohorts in which this question has been examined. Future studies with larger cohorts of patients with subtype specific hematologic cancers will help address this issue.

Vaccination is the most effective treatment for preventing SARS-CoV-2 infection and consequently prevention of COVID-19 associated mortality and morbidity. Unfortunately, patients with hematologic malignancies have decreased immunogenic responses to vaccines. This is more pronounced in patients with B-cell malignancies, those who have received anti-CD20 antibody therapy within 6 months, and CAR-T anti-CD19 and allo-SCT recipients [91]. However, a significant proportion of these patients still benefit from vaccination not only from a humoral antibody response but also from a CD8+ $\mathrm{T}$-cell response [39]. Therefore, the use of vaccines and boosters should be encouraged.

Studies conducted earlier in the pandemic reported a higher mortality rate with a relatively low ICU admission rate for patients with hematologic malignancies, suggesting issues with resource allocation at least early during the pandemic. Although patients with hematologic malignancies and COVID-19 have high overall mortality, they still benefit from the escalation of care when clinically indicated. Therefore, decisions regarding ICU admission for this patient population should still follow the standard of care clinical guidelines. Studies conducted later in the pandemic report improved outcomes in patients with COVID-19 and hematologic malignancies. This can likely be attributed to a better understanding of effective COVID-19-specific treatments, vaccination, and increased availability of resources.

Patients with hematologic malignancies might benefit from distinct COVID-19 directed therapies than patients in the general population. Unfortunately, these patients 
have been underrepresented in clinical trials of COVID-19 treatments. Despite negative results from randomized control trials for the use of convalescent plasma, retrospective data support this treatment for patients with COVID-19 and hematologic malignancies. Specifically, it appears that early use of this treatment might be beneficial. Similarly, the use of monoclonal antibodies has shown some promise. Large randomized controlled trials with higher recruitment of patients with hematologic malignancies and COVID-19 will help shed more light on this question. Additionally, cohort studies to assess how COVID-19 directed treatments modulate the immunogenic response to SARS-CoV-2 infection in patients with hematologic malignancies will be very informative.

The COVID-19 pandemic presents an evolving healthcare challenge to patients with and without cancer. However, patients with hematologic malignancies are particularly susceptible to worse outcomes. Therefore, efforts must be dedicated to a better understanding of the pathophysiology of SARS-CoV-2 infections in this immunocompromised population. The emergence of new variants threatens to erase some of the advances that have been made through vaccination and to lead to further surges with significant strains in the healthcare system. Clinical guidelines regarding treatment decisions should continue to evolve rapidly with the available information to best care for these patients. Therefore, updated and ongoing results from cohort studies in patients with cancer and COVID-19 are warranted.

\section{Conflict of Interest Statement}

The authors have no conflicts of interest to declare.

\section{Funding Sources}

This study did not receive any funding.

\section{Author Contributions}

J.C.M. reviewed the literature and wrote the review article. R.A.S. and K.S.-G reviewed the manuscript and made critical suggestions and modifications. S.M.R conceived and guided the study and wrote and critically reviewed the article for important intellectual content. All the authors approved the final version for submission.

\section{References}

1 WHO Coronavirus (COVID-19) Dashboard. WHO coronavirus (COVID-19) dashboard with vaccination data [Internet]. WHO; 2022 [cited 2022 Jan 24]. Available from: https:// covid19.who.int/.

2 Liang W, Guan W, Chen R, Wang W, Li J, Xu $\mathrm{K}$, et al. Cancer patients in SARS-CoV-2 infection: a nationwide analysis in China. Lancet Oncol. 2020;21(3):335-7.

3 Zhang L, Zhu F, Xie L, Wang C, Wang J, Chen $\mathrm{R}$, et al. Clinical characteristics of COVID19-infected cancer patients: a retrospective case study in three hospitals within Wuhan, China. Ann Oncol. 2020;31(7):894-901.

4 Dai M, Liu D, Liu M, Zhou F, Li G, Chen Z, et al. Patients with cancer appear more vulnerable to SARS-CoV-2: a multicenter study during the COVID-19 outbreak. Cancer Discov. 2020;10(6):783-91.

5 Mehta V, Goel S, Kabarriti R, Cole D, Goldfinger M, Acuna-Villaorduna A, et al. Case fatality rate of cancer patients with $\mathrm{CO}$ VID-19 in a New York hospital system. Cancer Discov. 2020;10(7):935-41.

6 Passamonti F, Cattaneo C, Arcaini L, Bruna $\mathrm{R}$, Cavo M, Merli F, et al. Clinical characteristics and risk factors associated with $\mathrm{CO}$ VID-19 severity in patients with haematological malignancies in Italy: a retrospective, multicentre, cohort study. Lancet Haematol. 2020;7(10): e737-45.

7 García-Suárez J, de la Cruz J, Cedillo Á, Llamas P, Duarte R, Jiménez-Yuste V, et al. Impact of hematologic malignancy and type of cancer therapy on COVID-19 severity and mortality: lessons from a large populationbased registry study. J Hematol Oncol. 2020; 13(1):133

8 Rubinstein SM, Warner JL. COVID-19 and haematological malignancy: navigating a narrow strait. Lancet Haematol. 2020;7(10): e701-3.

9 Desai A, Mohammed TJ, Duma N, Garassino MC, Hicks LK, Kuderer NM, et al. COVID-19 and cancer: a review of the registry-based pandemic response. JAMA Oncol. 2021; 7(12):1882-90.

10 Kuderer NM, Choueiri TK, Shah DP, Shyr Y, Rubinstein SM, Rivera DR, et al. Clinical impact of COVID-19 on patients with cancer (CCC19): a cohort study. Lancet. 2020; 395(10241):1907-18.

11 Desai A, Warner J, Kuderer N, Thompson M, Painter C, Lyman G, et al. Crowdsourcing a crisis response for COVID-19 in oncology. Nat Cancer. 2020;1(5):473-6.

12 Grivas P, Khaki AR, Wise-Draper TM, French B, Hennessy C, Hsu CY, et al. Association of clinical factors and recent anticancer therapy with COVID-19 severity among patients with cancer: a report from the COVID-19 and cancer consortium. Ann Oncol. 2021;32(6):787800 .

13 Lee LYW, Cazier JB, Starkey T, Briggs SEW, Arnold R, Bisht V, et al. COVID-19 prevalence and mortality in patients with cancer and the effect of primary tumour subtype and patient demographics: a prospective cohort study. Lancet Oncol. 2020;21(10):1309-16.

14 Pinato DJ, Zambelli A, Aguilar-Company J, Bower M, Sng CCT, Salazar R, et al. Clinical portrait of the SARS-CoV-2 epidemic in European patients with cancer. Cancer Discov. 2020;10(10):1465-74.

15 Jee J, Foote MB, Lumish M, Stonestrom AJ, Wills B, Narendra V, et al. Chemotherapy and COVID-19 outcomes in patients with cancer. J Clin Oncol. 2020;38(30):3538-46.

16 Pagano L, Salmanton-García J, Marchesi F, Busca A, Corradini P, Hoenigl M, et al. COVID-19 infection in adult patients with hematological malignancies: a European Hematology Association Survey (EPICOVIDEHA). J Hematol Oncol. 2021;14(1):168.

17 ASH RC COVID-19 [Internet]. [cited $2021 \mathrm{Oct}$ 21]. Available from: https://www.ashresearchcollaborative.org/s/covid-19-registry. 
18 Wood WA, Neuberg DS, Thompson JC, Tallman MS, Sekeres MA, Sehn LH, et al. Outcomes of patients with hematologic malignancies and COVID-19: a report from the ASH research collaborative data hub. Blood Adv. 2020;4(23):5966-75.

19 Nadkarni AR, Vijayakumaran SC, Gupta S, Divatia JV. Mortality in cancer patients with COVID-19 who are admitted to an ICU or who have severe COVID-19: a systematic review and meta-analysis. JCO Glob Oncol. 2021;7(7):1286-305

20 Regalado-Artamendi I, Jiménez-Ubieto A, Hernández-Rivas JÁ, Navarro B, Núñez L, Alaez C, et al. Risk factors and mortality of COVID-19 in patients with lymphoma: a Multicenter Study. Hemasphere. 2021;5(3): e538.

21 Vijenthira A, Gong IY, Fox TA, Booth S, Cook G, Fattizzo B, et al. Outcomes of patients with hematologic malignancies and COVID-19: a systematic review and meta-analysis of 3377 patients. Blood. 2020;136(25):2881-92.

22 Peckham H, de Gruijter NM, Raine C, Radziszewska A, Ciurtin C, Wedderburn LR, et al. Male sex identified by global COVID-19 meta-analysis as a risk factor for death and ITU admission. Nat Commun. 2020;11(1): 6317.

23 Piñana JL, Martino R, García-García I, Parody R, Morales MD, Benzo G, et al. Risk factors and outcome of COVID-19 in patients with hematological malignancies. Exp Hematol Oncol. 2020;9(1):21.

24 Tian J, Yuan X, Xiao J, Zhong Q, Yang C, Liu $\mathrm{B}$, et al. Clinical characteristics and risk factors associated with COVID-19 disease severity in patients with cancer in Wuhan, China: a multicentre, retrospective, cohort study. Lancet Oncol. 2020;21(7):893-903.

25 Albiges L, Foulon S, Bayle A, Gachot B, Pommeret F, Willekens $\mathrm{C}$, et al. Determinants of the outcomes of patients with cancer infected with SARS-CoV-2: results from the Gustave Roussy cohort. Nat Cancer. 2020;1(10):96575.

26 Chari A, Samur MK, Martinez-Lopez J, Cook G, Biran N, Yong K, et al. Clinical features associated with COVID-19 outcome in multiple myeloma: first results from the International Myeloma Society data set. Blood. 2020; 136(26):3033-40.

27 Mato AR, Roeker LE, Lamanna N, Allan JN, Leslie L, Pagel JM, et al. Outcomes of COVID-19 in patients with CLL: a multicenter international experience. Blood. 2020; 136(10):1134-43.

28 Mehta P, McAuley DF, Brown M, Sanchez E, Tattersall RS, Manson JJ, et al. COVID-19: consider cytokine storm syndromes and immunosuppression. Lancet. 2020;395(10229): 1033-4.

29 Valle DMD, Kim-Schulze S, Huang $\mathrm{HH}$, Beckmann ND, Nirenberg S, Wang B, et al. An inflammatory cytokine signature predicts COVID-19 severity and survival. Nat Med. 2020;26(10):1636-43.
30 Wynants L, Calster BV, Collins GS, Riley RD, Heinze G, Schuit E, et al. Prediction models for diagnosis and prognosis of covid-19: systematic review and critical appraisal. BMJ. 2020;369:m1328.

31 D'Aiello A, Zareef S, Pradhan K, Lombardo A, Khatun F, Mustafa J, et al. Older age and increased neutrophil-to-lymphocyte ratio (NLR) are predictors of mortality in a multiethnic urban cohort of hematologic neoplasms and COVID-19 patients. Blood. 2020; 136(Supplement 1):34-5.

32 Yancy CW. COVID-19 and African Americans. JAMA. 2020;323(19):1891-2.

33 Chowkwanyun M, Reed AL Jr. Racial health disparities and covid-19 - caution and context. N Engl J Med. 2020;383(3):201-3.

34 Mackey K, Ayers CK, Kondo KK, Saha S, Advani SM, Young S, et al. Racial and ethnic disparities in COVID-19: related infections, hospitalizations, and deaths: a systematic review. Ann Intern Med. 2021;174(3):362-73.

35 Schmidt AL, Bakouny Z, Bhalla S, Steinharter JA, Tremblay DA, Awad MM, et al. Cancer care disparities during the COVID-19 pandemic: COVID-19 and cancer Outcomes Study. Cancer Cell. 2020;38(6):769-70.

36 Rivera DR, Peters S, Panagiotou OA, Shah DP, Kuderer NM, Hsu CY, et al. Utilization of COVID-19 treatments and clinical outcomes among patients with cancer: a COVID-19 and cancer consortium (CCC19) Cohort Study. Cancer Discov. 2020;10(10):1514-27.

37 Hultcrantz M, Richter J, Rosenbaum CA, Patel D, Smith EL, Korde N, et al. COVID-19 infections and outcomes in patients with multiple myeloma in New York City: a cohort study from five academic centers. Blood Cancer Discov. 2020;1(3):234-43.

38 Bakouny Z, Hawley JE, Choueiri TK, Peters S, Rini BI, Warner JL, et al. COVID-19 and cancer: current challenges and perspectives. Cancer Cell. 2020;38(5):629-46.

39 Bange EM, Han NA, Wileyto P, Kim JY, Gouma S, Robinson J, et al. CD8+ T cells contribute to survival in patients with COVID-19 and hematologic cancer. Nat Med. 2021; 27(7):1280-9.

40 Knaus HA, Berglund S, Hackl H, Blackford AL, Zeidner JF, Montiel-Esparza R, et al. Signatures of $\mathrm{CD} 8+\mathrm{T}$ cell dysfunction in AML patients and their reversibility with response to chemotherapy. JCI Insight. 2018;3(21): e120974.

41 Nunes C, Wong R, Mason M, Fegan C, Man S, Pepper C. Expansion of a CD8+PD-1+ replicative senescence phenotype in early stage CLL patients is associated with inverted CD4: CD8 ratios and disease progression. Clin Cancer Res. 2012;18(3):678-87.

42 Zelle-Rieser C, Thangavadivel S, Biedermann R, Brunner A, Stoitzner P, Willenbacher E, et al. $T$ cells in multiple myeloma display features of exhaustion and senescence at the tumor site. J Hematol Oncol. 2016;9(1):116.
43 Moderbacher CR, Ramirez SI, Dan JM, Grifoni A, Hastie KM, Weiskopf D, et al. Antigen-specific adaptive immunity to SARSCoV-2 in acute COVID-19 and associations with age and disease severity. Cell. 2020; 183(4):996-1012.e19.

44 Shah GL, DeWolf S, Lee YJ, Tamari R, Dahi PB, Lavery JA, et al. Favorable outcomes of COVID-19 in recipients of hematopoietic cell transplantation. J Clin Invest. 2020;130(12): 6656-67.

45 Sharma A, Bhatt NS, Martin AS, Abid MB, Bloomquist J, Chemaly RF, et al. Clinical characteristics and outcomes of COVID-19 in haematopoietic stem-cell transplantation recipients: an observational cohort study. Lancet Haematol. 2021;8(3):e185-93.

46 Ljungman $\mathrm{P}$, de la Camara R, Mikulska M, Tridello G, Aguado B, Zahrani MA, et al. COVID-19 and stem cell transplantation; results from an EBMT and GETH multicenter prospective survey. Leukemia. 2021;35(10): 2885-94.

47 Busca A, Salmanton-García J, Corradini P, Marchesi F, Cabirta A, Blasi RD, et al. COVID-19 and CAR-T cells: current challenges and future directions-a report from the EPICOVIDEHA survey by EHA-IDWP. Blood Adv. 2021:bloodadvances.2021005616. Online ahead of print.

48 Lee LY, Cazier JB, Angelis V, Arnold R, Bisht $\mathrm{V}$, Campton NA, et al. COVID-19 mortality in patients with cancer on chemotherapy or other anticancer treatments: a prospective cohort study. Lancet. 2020;395(10241):1919-26.

49 Mossuto S, Attardi E, Alesiani F, Angelucci E, Balleari E, Bernardi M, et al. SARS-CoV-2 in myelodysplastic syndromes: a snapshot from early Italian experience. Hemasphere. 2020; 4(5):e483

50 Barbui T, Vannucchi AM, Alvarez-Larran A, Iurlo A, Masciulli A, Carobbio A, et al. High mortality rate in COVID-19 patients with myeloproliferative neoplasms after abrupt withdrawal of ruxolitinib. Leukemia. 2021; 35(2):485-93.

51 Scarfò L, Chatzikonstantinou T, Rigolin GM, Quaresmini G, Motta M, Vitale C, et al. COVID-19 severity and mortality in patients with chronic lymphocytic leukemia: a joint study by ERIC, the European Research Initiative on CLL, and CLL Campus. Leukemia. 2020;34(9):2354-63.

52 Yeleswaram S, Smith P, Burn T, Covington M, Juvekar A, Li Y, et al. Inhibition of cytokine signaling by ruxolitinib and implications for COVID-19 treatment. Clin Immunol. 2020;218:108517.

53 Roschewski M, Lionakis MS, Sharman JP, Roswarski J, Goy A, Monticelli MA, et al. Inhibition of Bruton tyrosine kinase in patients with severe COVID-19. Sci Immunol. 2020;5(48): eabd0110.

54 Paneesha S, Pratt G, Parry H, Moss P. Covid-19 infection in therapy-naive patients with B-cell chronic lymphocytic leukemia. Leuk Res. 2020;93:106366. 
55 Roeker LE, Eyre TA, Thompson MC, Lamanna N, Coltoff A, Davids MS, et al. COVID-19 in patients with CLL: improved survival outcomes and update on management strategies. Blood. 2021;138(18):1768-73.

56 Treon SP, Castillo JJ, Skarbnik AP, Soumerai JD, Ghobrial IM, Guerrera ML, et al. The BTK inhibitor ibrutinib may protect against pulmonary injury in COVID-19: infected patients. Blood. 2020;135(21):1912-5.

57 Group RC, Horby P, Lim WS, Emberson JR, Mafham M, Bell JL, et al. Dexamethasone in hospitalized patients with Covid-19. N Engl J Med. 2020;384(8):693-704.

58 Beigel JH, Tomashek KM, Dodd LE, Mehta AK, Zingman BS, Kalil AC, et al. Remdesivir for the treatment of Covid-19 - final report. N Engl J Med. 2020;383(19):1813-26.

59 Abani O, Abbas A, Abbas F, Abbas M, Abbasi $\mathrm{S}$, Abbass $\mathrm{H}$, et al. Tocilizumab in patients admitted to hospital with COVID-19 (RECOVERY): a randomised, controlled, open-label, platform trial. Lancet. 2021; 397(10285):1637-45.

60 RECOVERY Collaborative Group; Horby PW, Mafham M, Peto L, Campbell M, PessoaAmorim G, et al. Casirivimab and imdevimab in patients admitted to hospital with COVID-19 (RECOVERY): a randomised, controlled, open-label, platform trial. Medrxiv. 2021. Epub ahead of print.

61 Abaleke E, Abbas M, Abbasi S, Abbott A, Abdelaziz A, Abdelbadiee S, et al. Azithromycin in patients admitted to hospital with COVID-19 (RECOVERY): a randomised, controlled, open-label, platform trial. Lancet. 2021;397(10274):605-12.

62 RECOVERY Collaborative Group; Horby P, Mafham M, Linsell L, Bell JL, Staplin N, et al. Effect of hydroxychloroquine in hospitalized patients with Covid-19. N Engl J Med. 2020; 383(21):2030-40

63 RECOVERY Collaborative Group; Horby PW, Mafham M, Bell JL, Linsell L, Staplin N, et al. Lopinavir-ritonavir in patients admitted to hospital with COVID-19 (RECOVERY): a randomised, controlled, open-label, platform trial. Lancet. 2020;396(10259):1345-52.

64 Abani O, Abbas A, Abbas F, Abbas M, Abbasi S, Abbass $\mathrm{H}$, et al. Convalescent plasma in patients admitted to hospital with COVID-19 (RECOVERY): a randomised controlled, open-label, platform trial. Lancet. 2021;397(10289):2049-59.

65 Simonovich VA, Pratx LDB, Scibona P, Beruto MV, Vallone MG, Vázquez C, et al. A randomized trial of convalescent plasma in covid-19 severe pneumonia. N Engl J Med. 2020;384(7):619-29.

66 Korley FK, Durkalski-Mauldin V, Yeatts SD, Schulman K, Davenport RD, Dumont LJ, et al. Early convalescent plasma for high-risk outpatients with covid-19. N Engl J Med. 2021;385(21):1951-60.
67 Levy I, Lavi A, Zimran E, Grisariu S, Aumann S, Itchaki G, et al. COVID-19 among patients with hematological malignancies: a national Israeli retrospective analysis with special emphasis on treatment and outcome. Leuk Lymphoma. 2021;62(14):3384-93.

68 US Food and Administration. Coronavirus (COVID-19) update: FDA authorizes first oral antiviral for treatment of COVID-19 FDA [Internet]. US Food and Administration; 2021 [cited 2022 Jan 21]. Available from: https: //www.fda.gov/news-events/press-announcements/coronavirus-covid-19-updatefda-authorizes-first-oral-antiviral-treatment-covid-19.

69 Bernal AJ, Gomes da Silva MM, Musungaie DB, Kovalchuk E, Gonzalez A, Delos Reyes V, et al. Molnupiravir for oral treatment of covid-19 in nonhospitalized patients. N Engl J Med. 2022;386(6):509-20.

70 Passamonti F, Romano A, Salvini M, Merli F, Porta MGD, Bruna R, et al. COVID-19 elicits an impaired antibody response against SARS$\mathrm{CoV}-2$ in patients with haematological malignancies. Br J Haematol. 2021;195(3):371-7.

71 Thompson MA, Henderson JP, Shah PK, Rubinstein SM, Joyner MJ, Choueiri TK, et al. Association of convalescent plasma therapy with survival in patients with hematologic cancers and COVID-19. JAMA Oncol. 2021; 7(8):1167-75.

72 Klassen SA, Senefeld JW, Johnson PW, Carter RE, Wiggins CC, Shoham S, et al. The effect of convalescent plasma therapy on COVID-19 patient mortality: systematic review and meta-analysis. Mayo Clin Proc. 2021; 96(5):1262-75.

73 Kloypan C, Saesong M, Sangsuemoon J, Chantharit P, Mongkhon P. Convalescent plasma for COVID-19: a meta-analysis of clinical trials and real-world evidence. Eur J Clin Invest. 2021;51(11):e13663.

74 Piechotta V, Iannizzi C, Chai KL, Valk SJ, Kimber C, Dorando E, et al. Convalescent plasma or hyperimmune immunoglobulin for people with COVID-19: a living systematic review. Cochrane Database Syst Rev. 2021;5(5):CD013600

75 Janiaud P, Axfors C, Schmitt AM, Gloy V, Ebrahimi F, Hepprich M, et al. Association of convalescent plasma treatment with clinical outcomes in patients with COVID-19. JAMA. 2021;325(12):1185-95.

76 Puing AG, Ho S, Frankel P, Tegtmeier B, Martin A, Ross J, et al. SARS-CoV-2 specific monoclonal antibody for the treatment of mild-to-moderate COVID-19 in cancer patients: a single-center experience. J Infect Dis. 2022;225(2):352-4.

77 Razonable RR, Pawlowski C, O’Horo JC, Arndt LL, Arndt R, Bierle DM, et al. Casirivimab: imdevimab treatment is associated with reduced rates of hospitalization among high-risk patients with mild to moderate coronavirus disease-19. EClinicalMedicine. 2021;40:101102.
78 Le RQ, Li L, Yuan W, Shord SS, Nie L, Habtemariam BA, et al. FDA approval summary: tocilizumab for treatment of chimeric antigen receptor $\mathrm{T}$ cell-induced severe or lifethreatening cytokine release syndrome. Oncologist. 2018;23(8):943-7.

79 Hermine O, Mariette X, Tharaux PL, RescheRigon M, Porcher R, Ravaud P, et al. Effect of tocilizumab vs usual care in adults hospitalized with COVID-19 and moderate or severe pneumonia. JAMA Intern Med. 2021;181(1): 32-40.

80 REMAP-CAP Investigators; Gordon AC, Mouncey PR, Al-Beidh F, Rowan KM, Nichol $\mathrm{AD}$, et al. Interleukin- 6 receptor antagonists in critically ill patients with Covid-19. N Engl J Med. 2021;384(16):1491-502.

81 Rosas IO, Bräu N, Waters M, Go RC, Hunter $\mathrm{BD}$, Bhagani S, et al. Tocilizumab in hospitalized patients with severe covid-19 pneumonia. N Engl J Med. 2021;384(16):1503-16.

82 Stone JH, Frigault MJ, Serling-Boyd NJ, Fernandes AD, Harvey L, Foulkes AS, et al. Efficacy of tocilizumab in patients hospitalized with covid-19. N Engl J Med. 2020;383(24): 2333-44.

83 Group TWREA for C-19 T (REACT) W; Domingo P, Mur I, Mateo GM, del Gutierrez MM, Pomar V, et al. Association between administration of IL- 6 antagonists and mortality among patients hospitalized for COVID-19. JAMA. 2021;326(6):499-518.

84 Avni T, Leibovici L, Cohen I, Atamna A, Guz $\mathrm{D}$, Paul M, et al. Tocilizumab in the treatment of COVID-19 - a meta-analysis. QJM Int J Med. 2021;114(8):577-86

85 McDonald I, Murray SM, Reynolds CJ, Altmann DM, Boyton RJ. Comparative systematic review and meta-analysis of reactogenicity, immunogenicity and efficacy of vaccines against SARS-CoV-2. NPJ Vaccines. 2021; 6(1):74.

86 Griffiths EA, Segal BH. Immune responses to COVID-19 vaccines in patients with cancer: promising results and a note of caution. Cancer Cell. 2021;39(8):1045-7.

87 Greenberger LM, Saltzman LA, Senefeld JW, Johnson PW, DeGennaro LJ, Nichols GL. Antibody response to SARS-CoV-2 vaccines in patients with hematologic malignancies. Cancer Cell. 2021;39(8):1031-3.

88 Becerril-Gaitan A, Vaca-Cartagena BF, Ferrigno AS, Mesa-Chavez F, Barrientos-Gutiérrez $\mathrm{T}$, Tagliamento $\mathrm{M}$, et al. Immunogenicity and risk of SARS-CoV-2 infection after COVID-19 vaccination in patients with cancer: a systematic review and meta-analysis. Eur J Cancer. 2022;160:243-60.

89 Herishanu Y, Avivi I, Aharon A, Shefer G, Levi S, Bronstein Y, et al. Efficacy of the BNT162b2 mRNA COVID-19 vaccine in patients with chronic lymphocytic leukemia. Blood. 2021;137(23):3165-73. 
90 Greenberger LM, Saltzman LA, Senefeld JW, Johnson PW, DeGennaro LJ, Nichols GL. Anti-spike antibody response to SARS-CoV-2 booster vaccination in patients with $\mathrm{B}$ cellderived hematologic malignancies. Cancer Cell. 2021;39(10):1297-99.

91 Dhakal B, Abedin S, Fenske T, Chhabra S, Ledeboer N, Hari P, et al. Response to SARSCoV-2 vaccination in patients after hematopoietic cell transplantation and CAR T-cell therapy. Blood. 2021;138(14):1278-81.
92 Mairhofer M, Kausche L, Kaltenbrunner S, Ghanem R, Stegemann M, Klein K, et al. Humoral and cellular immune responses in SARS-CoV-2 mRNA-vaccinated patients with cancer. Cancer Cell. 2021;39(9):1171-2.

93 Pagano L, Salmanton-García J, Marchesi F, Lopez-Garcia A, Lamure S, Itri F, et al. COVID-19 in vaccinated adult patients with hematological malignancies. Preliminary results from EPICOVIDEHA. Blood. 2021: blood.2021014124. Epub ahead of print.
94 US Food and Administration. Coronavirus (COVID-19) update: FDA authorizes new long-acting monoclonal antibodies for preexposure prevention of COVID-19 in certain individuals FDA [Internet]. US Food and Administration; 2021 [cited 2022 Jan 21]. Available from: https://www.fda.gov/news-events/ press-announcements/coronavirus-covid19-update-fda-authorizes-new-long-actingmonoclonal-antibodies-pre-exposure. 